\title{
Pattern Formation Resulting from Faceted Growth in Zone-Melted Thin Films
}

\author{
Loren Pfeiffer, Scott Paine, ${ }^{(a)}$ G. H. Gilmer, Wim van Saarloos, and K. W. West \\ AT\&T Bell Laboratories, Murray Hill, New Jersey 07974 \\ (Received 31 October 1984)
}

\begin{abstract}
We develop a model for the recrystallization of Si films that are traversed by a molten zone. The model simulates the branching behavior of low-angle grain-boundary defects in these films to a remarkable degree. The simulated subboundary patterns scale approximately as the square root of the scan velocity, in excellent agreement with experiment.
\end{abstract}

PACS numbers: $68.55 .+\mathrm{b}, 68.30 .+\mathrm{z}$

In most studies of pattern formation in crystal growth diffusion of heat and diffusion of impurities are the dominant influences. The resulting cellular or dendritic structures have attracted much recent interest. $^{1,2}$ For an interface moving at speed $v$ with diffusion coefficient $D$, there are two relevant lengths: a diffusion length $D / v$, and a microscopic length $d_{0}$ which expresses the resistance of the interface to curvature. The resulting solidification patterns scale as $\lambda \simeq\left(d_{0} D / v\right)^{1 / 2}$, which decreases with increasing $v$.

We argue here on the other hand that the pattern of low-angle grain boundaries (subboundaries) in zonemelted silicon films is dominated by interface kinetics, and that as a result for this case $\lambda$ increases with $v$. Experimentally, it has indeed been found that $\lambda \propto v^{p}$, with $p=0.5$. Our model accounts for this and other properties of these low-angle grain-boundary patterns in a natural way. Moreover, while most models in the field of pattern formation that can be analyzed in some detail are only a crude approximation of the full problem, ${ }^{3}$ ours is not only simple but also appears to be realistic enough to compare directly to experimental data. As such, it also provides an interesting example of pattern formation that is a result of the competition in the growth of nearby facets, and of a fully deterministic nonlinear system that quickly settles statistically into a steady state.

In zone-melting recrystallization a hot zone is scanned at a constant speed $v$ over a thin film supported on a solid substrate. The film melts and then recrystallizes behind the heater. After recrystallization, the material is usually found to consist of a mosaic of regions, each of which has perfect crystalline order but whose crystalline orientation differs slightly from region to region. The resulting low-angle grain boundaries, or subboundaries, can be made visible by etching. Subboundary networks are often found in recrystallized metals ${ }^{4}$ such as $\mathrm{Cu}$ or $\mathrm{Sn}$, but have been most studied in thin $\mathrm{Si}$ films. For $\mathrm{Si}$ thin films essentially similar subboundary patterns are seen independently of the details of the melting, whether by incoherent light,${ }^{5}$ lasers, ${ }^{6}$ electric beams, ${ }^{7}$ or graphite strip heaters. ${ }^{8}$ Figure 1(a) is a Nomarski micrograph of a typical Si subboundary network obtained in our laboratory with use of graphite strip heater. ${ }^{9}$ The film con- sists of many $\mathrm{Si}(100)$ single crystals misoriented by typically $1^{\circ}$ to $2^{\circ}$ across each subboundary. Such experimental low-angle grain-boundary networks always show certain characteristics: (1) They run approximately parallel to the scan direction. (ii) Subboundaries that approach one another laterally appear to attract one another until they finally merge into a single survivor. (iii) New subboundaries are nucleated to maintain a constant average lateral density. (iv) Once nucleated, a subboundary does not terminate except by merging.

This work grew out of our study of the mechanism that causes adjacent nearby subboundaries to appear to be attracted to one another over long distances before finally merging. Rather than attributing this attraction to actual physical forces such as the long-range interac-

(a)

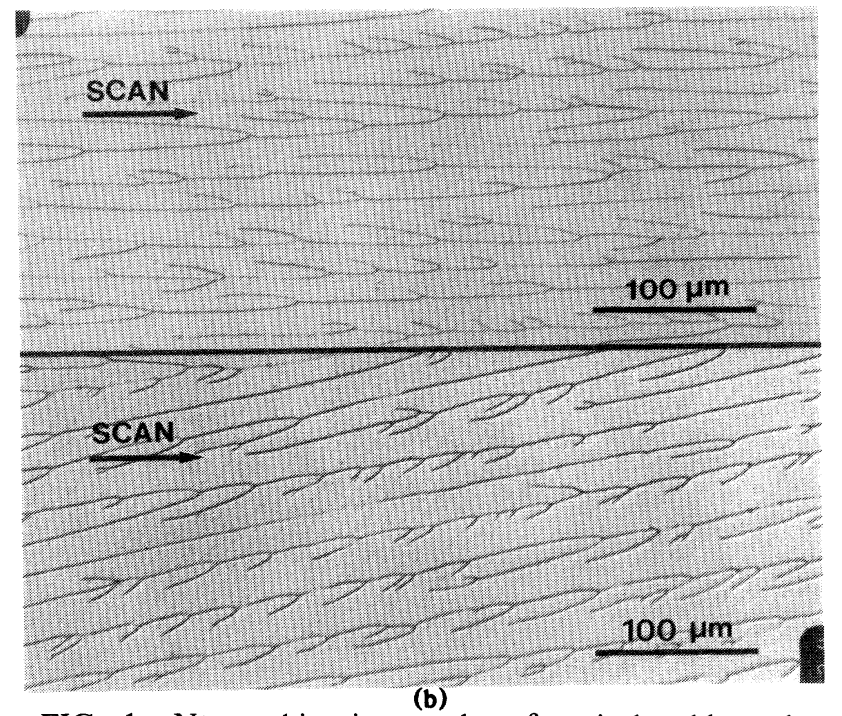

FIG. 1. Nomarski micrographs of typical subboundary networks. A $0.5-\mu \mathrm{m}$ polysilicon film between micron layers of amorphous $\mathrm{SiO}_{2}$ was recrystallized by scanning a narrow molten zone across the sample at $1.5 \mathrm{~mm} / \mathrm{sec}$. The cap oxide was then removed and a chemical defect etch was used to enhance the subboundaries. (a) Electron diffraction shows that this film has $(100)$ texture with $\langle 010\rangle$ along the scan. (b) Electron diffraction shows growth tilted by $7^{\circ}$ away from (100) texture and by $12^{\circ}$ away from the $\langle 010\rangle$ in-plane axis. 


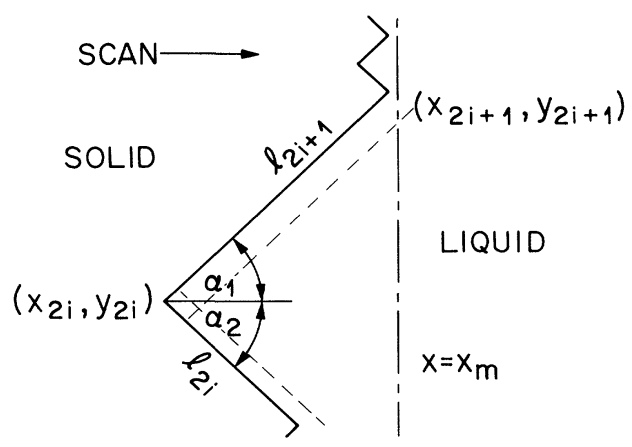

FIG. 2. Faceted melt-crystal interface (solid line). The dashed lines are a portion of this interface a short time later.

tion of dislocations, we have found that we can understand it quite naturally in terms of the motion of a faceted growth front.

The melt-solid interface of a growing Si crystal often establishes itself on the $\mathrm{Si}(111)$ crystal planes. ${ }^{10} \mathrm{Ex}-$ perimental zone-melted $\mathrm{Si}$ films on $\mathrm{SiO}_{2}$ tend to recrystallize with a (100) plane parallel to the film, that is with (100) texture, and with a $\langle 010\rangle$ axis along the direction of the scan. ${ }^{8}$ The growth interfaces of such films break up into facets defined by alternating (111) and $(11 \overline{1})$ planes. Geis and co-workers ${ }^{8,11}$ have obtained experimental evidence that subboundaries are formed at the interior corners [such as the one at $\left(x_{2 i}, y_{2 i}\right)$ in Fig. 2] of such a faceted front, and have proposed a qualitative model of subboundary formation. We have built upon their picture to develop a more quantitative understanding of the time evolution of subboundary networks. The detailed mechanism of subboundary formation is not yet understood. We have evidence, ${ }^{12,13}$ however, that it is due to the build up of excess oxygen in the melt which becomes further concentrated at reentrant corners in the growing solid.

We assume that the (111) and (111) facets at the growth interface advance by the nucleation of new layers, with each nucleation event contributing a complete new layer of atoms. Long facets tend to grow faster than short ones because they have more nucleation sites. This is sufficient to explain the apparent attraction of nearby subboundaries. To see this, consider Fig. 2. The solid line is the melt-solid interface of a Si film crystallizing with (100) texture along a $\langle 010\rangle$ growth axis. The position of the interface a moment later is drawn with a dashed line. Obviously, the corner at $\left(x_{2 i}, y_{2 i}\right)$ moves downward in time as a result of the fact that the long facet $l_{2 i+1}$ grows faster than the short one $l_{2 i}$. Thus, short facets shrink in size by being overtaken by long ones; this in turn causes subboundaries to merge in the way illustrated in Fig. 3, which shows the results of our simulations described below.

Our model assumes the following: (i) The scanning

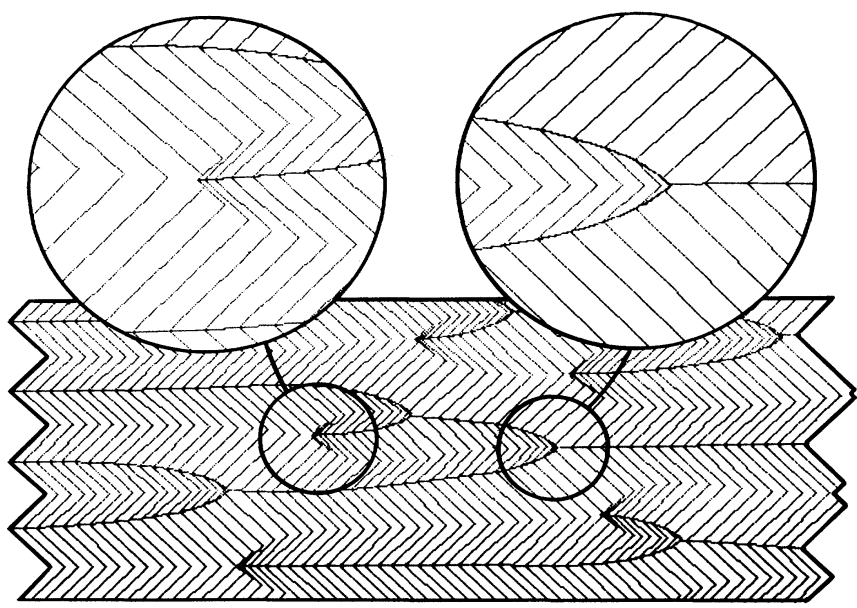

FIG. 3. Computer plot generated by Eqs. (1) and (2) showing the facets and the loci of the interior corners of the faceted growth front, moving from left to right.

strip heater or other heat source imposes a moving linear temperature gradient, parallel to the scan direction. (ii) The growth interface consists of alternating (111) and (111) facets, which may extend up to the melting isotherm, but not into the superheated region. (iii) The growth rate of each facet is limited only by the nucleation rate of new monolayers, and is independent of the spreading rate of the monolayers. (iv) The nucleation rate is zero at the melting isotherm, and increases linearly with the undercooling. ${ }^{14}$

Since all angles are fixed, the geometrical construction of Fig. 2 shows that the rate of change of the length $l_{2 i}$ of the 2 th facet is proportional to the difference in the normal growth rate of neighboring facets, or

$$
\begin{aligned}
& \frac{d}{d t} l_{2 i}=\frac{1}{\sin \left(\alpha_{1}+\alpha_{2}\right)}\left(R_{2 i-1}-R_{2 i+1}\right), \\
& \frac{d}{d t} l_{2 i+1}=\frac{1}{\sin \left(\alpha_{1}+\alpha_{2}\right)}\left(R_{2 i+2}-R_{2 i}\right),
\end{aligned}
$$

where $R_{i}$ is the normal growth rate of the ith facet. If the heat source is scanned at a velocity $v$, and $x_{m}$ is the position of the melting isotherm, then $x_{m}=v t$. By our assumptions it follows that the growth rate of a facet is an explicit function of its length and its position with respect to the melting isotherm, i.e.,

$$
\begin{aligned}
R_{2 i} & =\beta_{2} \int_{x_{2 i}}^{x_{2 i-1}} \frac{\left(x_{m}-x\right) d x}{\cos \alpha_{2}} \\
& =\beta_{2} l_{2 i}\left(x_{m}-\frac{x_{2 i+1}+x_{2 i}}{2}\right),
\end{aligned}
$$

and similarly,

$$
R_{2 i+1}=\beta_{1} l_{2 i+1}\left(x_{m}-\frac{x_{2 i+1}+x_{2 i}}{2}\right) .
$$

Here $\beta_{1}$ and $\beta_{2}$ are kinetic factors determined by the 
tilt between the plane of the Si film and the growing (111) and (111) facets. For films of (100) texture, $\beta_{1}=\beta_{2}$.

Plots of the dynamics of the facet growth were made by integration of Eqs. (1) and (2) numerically using a Runge-Kutta technique. Periodic boundary conditions were used to eliminate edge effects. The rule for generation of new facets was based on the mechanism described by Geis et al. ${ }^{11}$ If a peak formed by two adjacent facets approaches the melting point, it flattens and indents forming new (111) and (11i) facets. Initial indentations of only $10^{-5} l_{2 i}$ are sufficient for stable growth.

Figure 3 is a plot generated by the model showing the time development of the facets and the loci of the interior corners [e.g., point $\left(x_{2 i}, y_{2 i}\right)$ in Fig. 2] of the faceted growth front. The growth is along $\langle 010\rangle$ with no tilt, and thus $\alpha_{1}=\alpha_{2}=\pi / 4$, and $\beta_{1}=\beta_{2}$. Initially, all facets had equal length except for a $5 \%$ perturbation of one. The perturbation propagates rapidly, and the system quickly settles into a quasistable pattern of facet generation and annihilation. 15

The insets show interior-corner nucleation and annihilation in detail. Interior corners newly formed at $x_{m}$ recede towards lower-temperature isotherms, because they are bounded by short facets which grow more slowly than the longer facets of the second neighbors on both sides. For $\alpha_{1}=\alpha_{2}, \beta_{1}=\beta_{2}$, interior corners are always drawn toward the side with the shorter facet, so that neighboring pairs come together more and more rapidly as their separation decreases. While the model does not explicitly require that adjacent interior corners annihilate in pairs, they nearly always do so, because the dependence of the growth rate on undercooling tends to drive interior corners to a common temperature isotherm.

Figure 4 shows plots of the loci of interior corners of the faceted interface under several simulated growth conditions. Note the striking similarities to the experimental subboundary networks of Fig. 1. This harmony between simulation and experiment provides strong evidence for the proposition that subboundaries indeed originate at the interior corners of a faceted growth front.

The initial facet lengths differ by about a factor of 3 between Figs. 4(a) and 4(b). After a short evolution, however, the two patterns become very similar, and remain so until the simulated scan velocity was changed halfway across 4(b). This illustrates that faceted growth quickly establishes a pattern that, although continuing to change, is statistically a steady state. $^{15}$

Halfway across plot 4(b) the scan velocity, $v$, was reduced to $v / 4$. The overall scale of the pattern is reduced by almost a factor of 2 , but the aspect ratio and the general characteristics are otherwise unchanged. Variation of the parameter $\beta$ also affects only the scale (a)

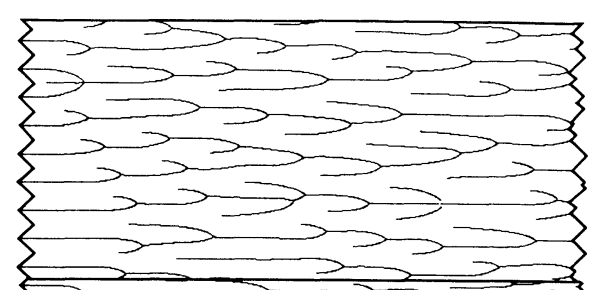

(b)

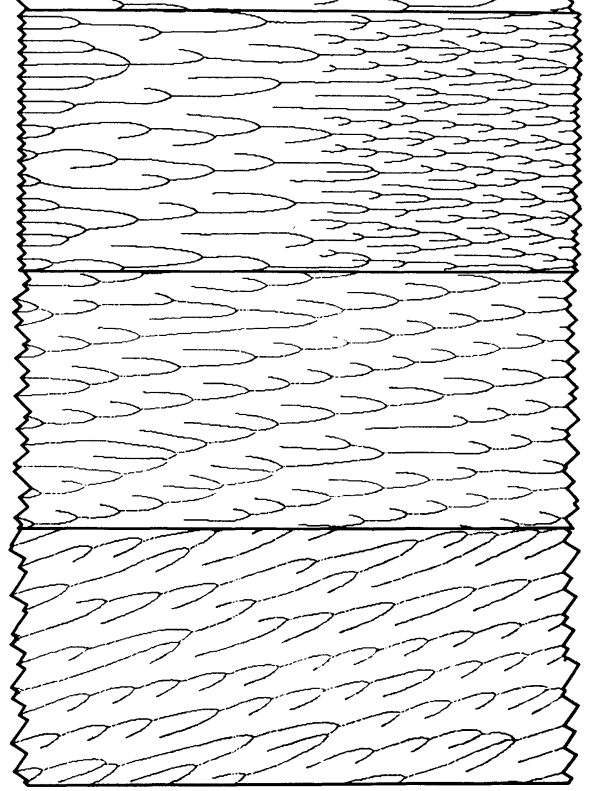

FIG. 4. Computer-generated plots of the loci of interior corners (referred to the solid) of the faceted melt front during growth. (a),(b) $\alpha_{1}=\alpha_{2}=45^{\circ}$ and $\beta_{1}=\beta_{2}$. Halfway across plot (b) the scan velocity was reduced from $v$ to $v / 4$. (c) $\alpha_{1}=41.5^{\circ}, \alpha_{2}=48.5^{\circ}, \beta_{1}=\beta_{2}$. (d) $\alpha_{1}=57^{\circ}, \alpha_{2}=33^{\circ}$, $\beta_{1}=1.9 \beta_{2}$.

of the pattern. This shows that the characteristics of the $\langle 010\rangle$ simulations are built into Eqs. (1) and (2) and, except for adjustments to the overall scale, there are no adjustable parameters.

At very large scan velocities we can expect a change in the nature of the growth process. With less time available for a nucleation cluster to spread laterally and cover the facet, other nuclei may be generated in the layer. In this case the growth rate will be less sensitive to the length of the facet. Indeed, experimental subboundary structures formed at high scan velocities tend to have fewer branches, indicating that one is approaching conditions where facet lengths are no longer affecting the kinetics. Large step velocities are implied in any case, since the time required for the step to move across the facet must be comparable to the average time between nucleation events. From the experimental data, a step velocity of several meters per second is obtained if we assume that these times are equal, and that there is only one nucleation event per layer.

The relation of scan velocity to pattern scale is of 
particular interest because it illustrates another point of close agreement between our simulations and experiment. Geis et al. ${ }^{11}$ have reported that for (100) $\mathrm{Si}$ films, the average distance $\lambda$ between subboundaries varies approximately as the square root of scan speed. We have confirmed this result for films grown in our laboratory, and find, as illustrated in Fig. 4(b), that the average distance between facet tracks in our simulations also shows this dependence. . One can understand this as follows: The forward corner of a typical facet pair will be quite close to the melting isotherm, so that $x_{2 i-1} \simeq x_{m}$. Substitution of this in Eq. (2) yields $R_{2 i} \simeq \frac{1}{2} \beta l_{2 i}^{2} \cos \alpha_{2}$. Since the growth rate of a typical facet is proportional to $v$, we get $l_{2 i} \propto v^{1 / 2}$. As noted above, models based on thermal or impurity diffusion effects cannot account even qualitatively for this dependence.

The more general cases of faceted growth along different axes (not exactly along the $\langle 010\rangle$ direction) and tilted away from (100) texture are shown in Figs. 4(c) and $4(\mathrm{~d})$, together with our experimental film that matches these conditions in Fig. 1(b). In experimental films we find that off-axis growth, with $\alpha_{1} \neq \alpha_{2}$, is most often also tilted off (100) texture so that $\beta_{1} \neq \beta_{2}$. Our model does not require this linkage, but can readily accommodate it. Consider simulation 4(c) with $\alpha_{1} \neq \alpha_{2}$ and $\beta_{1}=\beta_{2}$. Note that the branching is like the experimental subboundary pattern $1(\mathrm{~b})$, but from the wrong side. To get the branching to come from the other side we find that we must set $\alpha_{1}$ and $\alpha_{2}$ to agree with experiment as well as separately adjust $\beta_{1}$ and $\beta_{2}$ as was done in simulation $4(\mathrm{~d})$. Electron diffraction channeling of the film of Fig. 1(b) confirms that its texture is indeed tilted away from (100) by $7^{\circ}$.

We conclude there is a close correspondence between the loci of interior corners of the (111), (11 $\overline{1})$ faceted growth front, and the patterns formed by experimental low-angle grain boundaries in meltrecrystallized thin $\mathrm{Si}$ films. It is remarkable how well the patterns generated by the model and experiment agree. As mentioned previously these calculations do not specify the details of the subboundary formation at the interior corners. Indeed, we note that nothing in this work demands that subboundaries must form on the interior corners of the faceted front. It is quite possible to envision a dislocation-free single-crystal film growing from a melt of high purity with a faceted growth front but without subboundary formation.

The authors are pleased to thank David Joy for providing the electron diffraction channeling measurements, and John Poate and Ken Jackson for helpful discussions.

(a) Permanent address: Massachusetts Institute of Technology, Cambridge, Mass. 02139.

1J. S. Langer, Rev. Mod. Phys. 52, 1 (1980).
2R. F. Sekerka, Physica (Utrecht) D12, 212 (1984), and in more detail in "Encyclopedia of Materials Science and Engineering"' (Pergamon, Oxford, to be published).

${ }^{3}$ The patterns generated by our model (Fig. 4) superficially resemble some obtained by V. Datye, R. Mathur, and J. S. Langer, J. Stat. Phys. 29, 1 (1982), in what they call a "caricature of eutectic solidification." Indeed the mathematical structure of their model has some features in common with ours; however, the physics underlying our model is quite different from the physics of eutectic growth which is based on diffusion effects so that, as explained in the introduction, the scale of eutectic patterns decreases with velocity. Moreover, in our model the annihilation and creation of subboundaries occurs as a natural consequence of the underlying physics, whereas in the model of Datye, Mathur, and Langer an additional rule for the termination and creation of lamellae must be specified.

${ }^{4}$ K. A. Jackson, in Metals Handbook, edited by Taylor Lyman (American Society for Metals, Cleveland, Ohio, 1973), Vol. 8, 8th Ed., pp. 147-149.

${ }^{5}$ T. J. Stultz and J. F. Gibbons, Appl. Phys. Lett. 41, 824 (1982).

${ }^{6}$ A. Gat, L. Gerzberg, J. F. Gibbons, T. J. Magee, J. Peng, and D. Hong, Appl. Phys. Lett. 33, 775 (1978).

7J. R. Davis, R. A. McMahon, and H. Ahmed, in LaserSolid Interactions and Transient Thermal Processing of Materials, edited by J. Narayan, W. L. Brown, and R. A. Lemons (North-Holland, New York, 1983), p. 563.

${ }^{8}$ M. W. Geis, H. I. Smith, B.-Y. Tsaur, J. C. C. Fan, D. J. Silversmith, and R. W. Mountain, J. Electrochem. Soc. 129, 2813 (1982).

${ }^{9}$ L. Pfeiffer, J. M. Gibson, and T. Kovacs, in Thin Films and Interfaces II, edited by J. E. E. Baglin, D. R. Campbell, and W. K. Chu (North-Holland, New York, 1984), p. 505.

${ }_{10}$ Slow growth in small temperature gradients produces an interface bounded by $\{111\}$ planes with highly anisotropic growth kinetics. See G. H. Gilmer, in Laser-Solid Interactions and Transient Thermal Processing of Materials, edited by J. Narayan, W. L. Brown, and R. A. Lemons (NorthHolland, New York, 1983), p. 249. Surfaces other than \{111\} that may be initially present rapidly move out until only the slow-moving surfaces remain.

${ }^{11}$ M. W. Geis, H. I. Smith, D. J. Silversmith, R. W. Mountain, and C. V. Thompson, J. Electrochem. Soc. 130, 1178 (1983).

${ }^{12}$ L. Pfeiffer, G. K. Celler, T. Kovacs, and McD. Robinson, Appl. Phys. Lett. 43, 1048 (1983).

${ }^{13}$ L. Pfeiffer, K. W. West, and T. Kovacs, to be published. ${ }^{14}$ Two-dimensional nucleation theory suggests a more complex relationship between the nucleation rate and undercooling. See J. D. Weeks and G. H. Gilmer, Adv. Chem. Phys. 40, 157 (1979). In our case nucleation is affected by the $\mathrm{SiO}_{2}: \mathrm{Si}$ interfaces, and the kinetics are not precisely defined. Although the qualitative features of the model will not be changed by the use of more faithful expressions for the nucleation kinetics, the value of $p$ in the scale factor $\lambda$ will be affected somewhat.

${ }^{15}$ Although our model is fully deterministic, it exhibits the "sensitive dependence on initial conditions" often found in nonlinear dynamics. As a result, it quickly settles into a statistically steady state, independent of the starting configuration. 
(a)

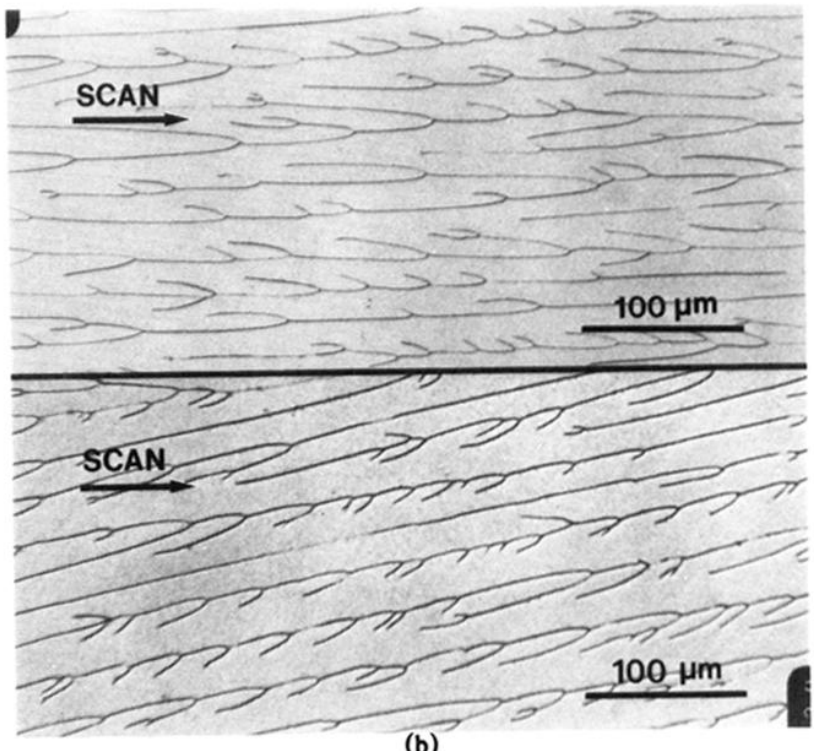

(b)

FIG. 1. Nomarski micrographs of typical subboundary networks. A $0.5-\mu \mathrm{m}$ polysilicon film between micron layers of amorphous $\mathrm{SiO}_{2}$ was recrystallized by scanning a narrow molten zone across the sample at $1.5 \mathrm{~mm} / \mathrm{sec}$. The cap oxide was then removed and a chemical defect etch was used to enhance the subboundaries. (a) Electron diffraction shows that this film has (100) texture with $\langle 010\rangle$ along the scan. (b) Electron diffraction shows growth tilted by $7^{\circ}$ away from (100) texture and by $12^{\circ}$ away from the $\langle 010\rangle$ in-plane axis. 